\title{
Personnel Ranking and Selection Problem Solution by Application of KEMIRA Method
}

\author{
N. Kosareva, E.K. Zavadskas, A. Krylovas, S. Dadelo
}

\author{
Natalja Kosareva, Edmundas Kazimieras Zavadskas*, \\ Aleksandras Krylovas, Stanislav Dadelo \\ Vilnius Gediminas Technical University \\ Sauletekio al. 11, LT-10223 Vilnius, Lithuania \\ natalja.kosareva@vgtu.lt, edmundas.zavadskas@vgtu.lt \\ aleksandras.krylovas@vgtu.lt,stanislav.dadelo@vgtu.lt \\ *Corresponding author: edmundas.zavadskas@vgtu.lt
}

\begin{abstract}
In this study KEmeny Median Indicator Rank Accordance (KEMIRA) method is applied for solving personnel ranking and selection problem when there are two subgroups of evaluating criteria. Each stage of KEMIRA method illustrated with the examples. In the first stage Kemeny median method is applied to generalize experts' opinions for setting criteria priorities. Medians were calculated for all experts opinions generalization and for experts majority opinions generalization. In the second stage criteria weights calculated and alternatives ranking accomplished simultaneously by Indicator Rank Accordance method. The obtained solutions compared with the results received in previous work of authors.
\end{abstract}

Keywords: multiple criteria decision making, Kemeny median, criteria priority, optimization problem.

\section{Introduction}

Personnel ranking and selection process in companies is focused on testing and evaluating of human resource potential, skills and personal characteristics. Developed personnel selection and evaluation systems are usually oriented to companies operating cost reduction in order to optimize human resources demand and layout planning [8]. Personnel selection and placement of the necessary positions is seen as the most important factor affecting organization's security, stability and development [20]. Proper recruitment has influence on organization's climate directly or influencing it through mediators [34]. Security personnel selection process requires identification of specific criteria for the occupied position and setting of their weights values [12]. Solution of this problem is relevant to many companies and organizations. At the same time it is necessary to create unified (being in accordance with uniform standards) personnel selection algorithms [3]. Personnel selection deployment process is directed to human resource potential, skills and personal features, i.e. factors affecting professional efficiency, testing and evaluation. Research has revealed that the main factors affecting the professional ability to work are of different origin: physical, psychological, cognitive, social/behavioral, workplace factors and factors outside the workplace [18]. This investigation has not determined the strength (weight) of identified factors influence on professional working capacity. It can be assumed that these factors have a different impact on the effectiveness of different professional activities. Experts' assessment applied for weight identification is often a subjective process based on stereotypes, attitudes, sympathy and so on [15]. The results of such evaluation often do not meet the expectations of the ultimate goal. From the other hand application of specialized tests (objective assessments) for weight identification meets the uncertainty factor. It is not clear how much test results will be concerned with the quality of the work results in the future [22]. According to the authors the most reliable methodology of professional selection is integrating several fields of criteria 
and sub-criteria systems [41]. It was found that data obtained with the objective (internal) and subjective (external) methods correlate with each other at about 0.3 [11], so harmonization of objective and subjective criteria schemes allow to expect a higher efficiency of the staff selection process [13].

Solution of this relevant problem is often subject to the Multiple Criteria Decision Making methods (MCDM). MCDM is rapidly evolving methodologies direction, does have considerable researchers attention. Decision-making methods face new requirements for modernization. There is a variety of MCDM methods. A monograph [39] provides readers with a capsule look into the existing methods, their characteristics and applicability to analysis of Multiple Attribute Decision Making (MADM) problems. Models for MADM, transformation of attributes, fuzzy decision rules, and methods for assessing weights are presented in this work. The paper [42] presents a panorama of decision making methods in economics and summarizes the most important results and applications which have appeared in the last decade. [33] added several new important concepts and trends in the MCDM field for solving actual problems. They provided comments on a previously published paper of [42] that could be thought of as an attempt to complete the original paper. Despite the intensive development worldwide, few attempts have been made to systematically present the theoretical bases and developments of MCDM methods. The article [43] describes the situation with reviews of MCDM/MADM methods. The goal of the paper [31] is to propose an approach to resolve disagreements among MCDM methods based on Spearman's rank correlation coefficient. The authors showed that the proposed approach can resolve conflicting MCDM rankings and reach an agreement among different MCDM methods. Sometimes, there are situations where MCDM methods generate very different results. The results proposed in [35] proved that MCDM methods are useful tools for evaluating multiclass classification algorithms and the fusion approach of MCDM methods is capable of identifying a compromised solution when different MCDM methods generate conflicting rankings.

However, MCDM techniques for recruitment and layout planning areas are not consistently structured and improved, this is an obstacle to their wide application. New MCDM methods are developing and new fields are looking for their application. The fuzzy MULTIMOORA for group decision making (MULTIMOORA-FG) enables to aggregate subjective assessments of the decision-makers and offer an opportunity to perform more robust personnel selection procedures [4], [5]. The selection process often contains imprecise data and linguistic variables. The model proposed in [6] combines fuzzy analytic hierarchy process (FAHP) and Technique for Order Preference by Similarity to Ideal Solution (TOPSIS) approaches to overcome these problems. The personnel selection problem is suitable to be dealt with by the linguistic VIKOR method because it includes some conflicting criteria and needs to consider the relative competitiveness of each candidate [9], [16]. The hybrid MCDM models which employ analytic network process (ANP) and modified TOPSIS and combination of the fuzzy Delphi method, ANP, and TOPSIS demonstrated the effectiveness and feasibility of the proposed models for personnel selection [14] and for investment in stock exchange [19]. The model based on fuzzy DEMATEL (Decision Making Trial and Evaluation Laboratory) and fuzzy ANP is proposed in [25] to to cope with the interdependencies between evaluation criteria. In the mentioned paper, a fuzzy DEMATEL-ANP model is proposed for selection of snipers. An approach enabling combination of Fuzzy ANP, Fuzzy TOPSIS and Fuzzy ELECTRE techniques were enabled for solving of the same problem [26]. Other authors proposed other MCDM methods, like fuzzy AHP adopted for Triangular Fuzzy Numbers (TFN) [37] or such as ELECTRE III and PROMETHEE II [27], [36] in the process of staff selection and recruitment.

Other methods used for solving similar problems are grey relational analysis (GRA) based methods [41]. A GRA-based intuitionistic fuzzy multi-criteria group decision making method for personnel selection is proposed in [23]. In this paper intuitionistic fuzzy weighted averaging 
(IFWA) operator is utilized to aggregate individual opinions of decision makers and intuitionistic fuzzy entropy is used to obtain the entropy weights of the criteria.

The hybrid approach applying AHP and TOPSIS grey as an effective decision aid to improve human resources management in various areas of economic activities is proposed in [23]. A personnel selection system based AHP and Complex proportional assessment of alternatives with grey relations (COPRAS-G) method was proposed in [24]. The use of hesitant fuzzy sets as a powerful technique has been studied in [20]. This paper explores aggregation methods for prioritized hesitant fuzzy elements and their application on personnel evaluation.

The comparison of various methods in the selection of personnel may help in finding out the accuracy, appropriateness, suitability, fairness and practicality efficiently [37]. Using several independent sub-criteria for personnel selection we face with the lack of appropriate MCDM solutions. The purpose of this article is to offer methods of this problem solution.

Studies have shown that the subjective and objective assessments are different [2], [21]. In order to effective solutions, it is necessary to synthesize the objective and subjective assessments, this opens up new opportunities and improve the quality of the selection process [10]. Most previous studies did not provide clear criteria for grouping and ranking process patterns [30]. The proposed new KEMIRA method allows to combine criteria structuring and evaluating on the basis of subjective and objective levels. This method can be used to solve unstructured evaluation tasks when consensus among the experts on the importance of sub-criteria is not obligatory or when criteria of different nature (genesis) are used. It occurs in those areas of human activity where it is necessary the evaluation of experts from different specializations (personnel, finance, manufacturing and technology, etc.). Using this algorithm for decision-making, the decision-making process takes on a higher quality and greater integration levels to include in the evaluation and selection process a lot of important criteria of different nature, incomparable among themselves and couldn't be combined. For example, external and internal criteria; subjective and objective criteria; physical, mental and psychological indicators, etc. The proposed KEMIRA criteria selection and structuring model extends the MCDM approach. The advantage of proposed KEMIRA method is its efficiency for solving MCDM problems when the set of criteria is divided into a few subgroups having different origin. Nevertheless, Kemeny median method, which is a part of KEMIRA method, is applicable for much wider class of MCDM problems when it is necessary to establish priority of criteria. It must be mentioned that criteria weights are found at the same time as the process of MCDM problem solution (ranking the alternatives) goes on.

\section{Problem formulation and scheme of solution}

This work continues the series of works designed to solve MCDM problem of elite selection from security personnel, when two separate groups of criteria describing security guards are presented. In general case it may be more than two groups of criteria. External assessment values and internal measurements are given for each subject. Additionally, information about experts independently set preferences for criteria in each criterion group is known. The purpose of the study is selection of $10 \%$ best subjects according to the given information. Usually MCDM problems are being solved in the following order. At first stage of the problem solution data normalization procedure is performed. Then criteria weights are determined and finally MCDM methods applied for selecting best objects and/or objects ranking [29]. Each problem solution phase can be performed by different methods.

Our proposed KEmeny Median Indicator Rank Accordance (KEMIRA) [32] method consists of other three phases:

1. data normalization and standardization;

2. determination of criteria preferences reflecting the aggregated experts opinion; 
3. minimization of the target functions value, determination of criteria weights and objects ranking.

The second step is determination priority of criteria by Kemeny median method. In this stage we'll use the information about criteria preferences set by experts. Note that the third step combines two phases into one - determination of criteria weights and objects ranking will be carried out simultaneously. This process is dynamic, so both goals will be achieved at the end of optimization procedure. Brief scheme of problem solution is depicted in Figure 1.

Comprehensive description of the method steps is provided below. At first introduce some notations: $m$-number of internal criteria $(X), n$ - number of external criteria $(Y), K$-number of alternatives, $S$ - number of experts. Initial decision-making matrix:

$$
D=\left(\begin{array}{cccccccccc}
x_{1}^{(1)} & \ldots & x_{j}^{(1)} & \ldots & x_{n}^{(1)} & y_{1}^{(1)} & \ldots & y_{j}^{(1)} & \ldots & y_{m}^{(1)} \\
\ldots & \ldots & \ldots & \ldots & \ldots & \ldots & \ldots & \ldots & \ldots & \ldots \\
x_{1}^{(i)} & \ldots & x_{j}^{(i)} & \ldots & x_{n}^{(i)} & y_{1}^{(i)} & \ldots & y_{j}^{(i)} & \ldots & y_{m}^{(i)} \\
\ldots & \ldots & \ldots & \ldots & \ldots & \ldots & \ldots & \ldots & \ldots & \ldots \\
x_{1}^{(K)} & \ldots & x_{j}^{(K)} & \ldots & x_{n}^{(K)} & y_{1}^{(K)} & \ldots & y_{j}^{(K)} & \ldots & y_{m}^{(K)}
\end{array}\right)
$$

Criteria preferences estimated by experts:

\begin{tabular}{|c|ccccc|ccccc|}
\hline Expert & $x_{1}$ & $\ldots$ & $x_{l}$ & $\ldots$ & $x_{n}$ & $y_{1}$ & $\ldots$ & $y_{l}$ & $\ldots$ & $y_{m}$ \\
\hline 1 & $i_{1}^{(1)}$ & $\ldots$ & $i_{l}^{(1)}$ & $\ldots$ & $i_{n}^{(1)}$ & $j_{1}^{(1)}$ & $\ldots$ & $j_{l}^{(1)}$ & $\ldots$ & $j_{m}^{(1)}$ \\
$\ldots$ & $\ldots$ & $\ldots$ & $\ldots$ & $\ldots$ & $\ldots$ & $\ldots$ & $\ldots$ & $\ldots$ & $\ldots$ & $\ldots$ \\
$\mathrm{s}$ & $i_{1}^{(s)}$ & $\ldots$ & $i_{l}^{(s)}$ & $\ldots$ & $i_{n}^{(s)}$ & $j_{1}^{(s)}$ & $\ldots$ & $j_{l}^{(s)}$ & $\ldots$ & $j_{m}^{(s)}$ \\
$\ldots$ & $\ldots$ & $\ldots$ & $\ldots$ & $\ldots$ & $\ldots$ & $\ldots$ & $\ldots$ & $\ldots$ & $\ldots$ & $\ldots$ \\
$\mathrm{S}$ & $i_{1}^{(S)}$ & $\ldots$ & $i_{l}^{(S)}$ & $\ldots$ & $i_{n}^{(S)}$ & $j_{1}^{(S)}$ & $\ldots$ & $j_{l}^{(S)}$ & $\ldots$ & $j_{m}^{(S)}$ \\
\hline
\end{tabular}

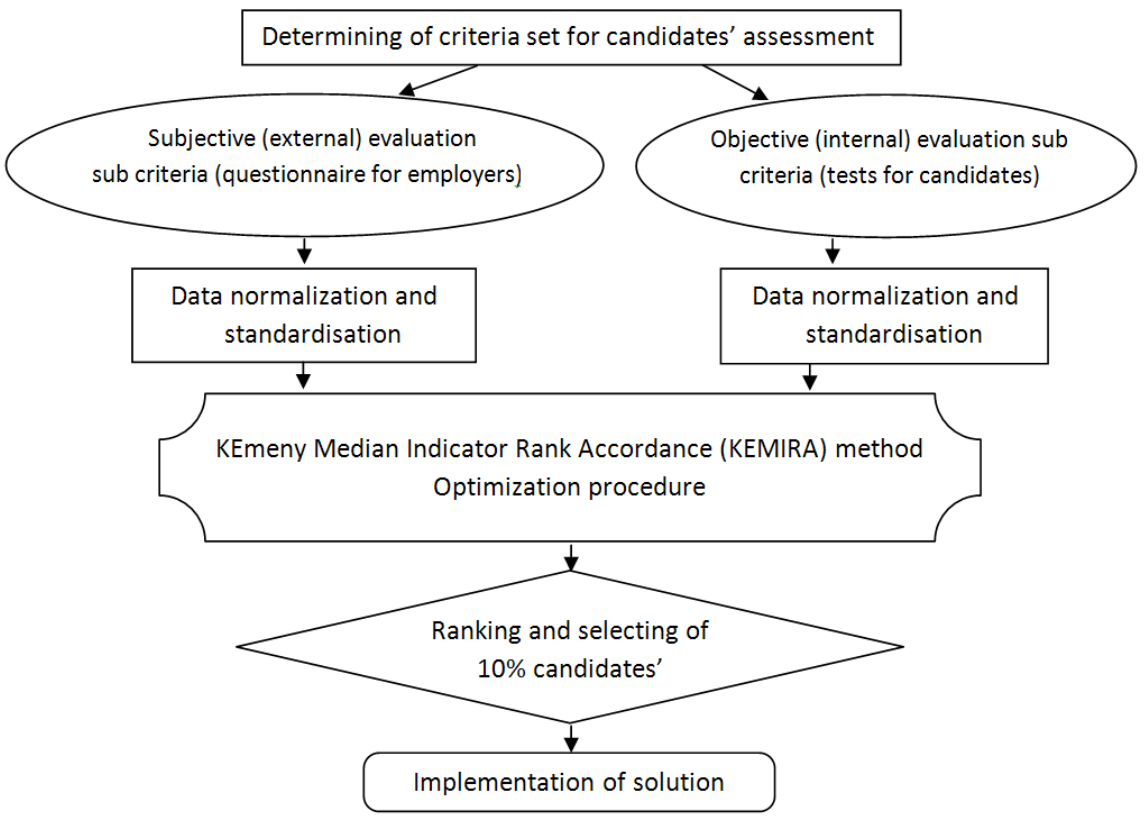

Figure 1: Scheme of KEmeny Median Indicator Rank Accordance Method (KEMIRA) application for selecting the best objects.

Step 1. Normalization of elements of decision making matrix (1). All criteria are representing a benefit, i.e. the bigger is a value, the better is the respective alternative. Elements of decision- 
making matrix are normalized by formulas:

$$
x_{j}^{(i) *}=\left(x_{j}^{(i)}-x_{\min }^{(i)}\right) /\left(x_{\max }^{(i)}-x_{\min }^{(i)}\right), \quad y_{j}^{(i) *}=\left(y_{j}^{(i)}-y_{\min }^{(i)}\right) /\left(y_{\max }^{(i)}-y_{\min }^{(i)}\right) .
$$

Step 2. Determining priority of criteria $X$ and $Y$ components. Criteria $X$ and $Y$ components priority is established independently by choosing priority which minimizes sum of distances to the priorities set up by all $S$ experts:

$$
R_{A}=\arg \min _{R} \sum_{j=1}^{S} \rho_{A}\left(R, R^{(j)}\right) .
$$

The result of this step is the median criteria components priority: $x_{j_{1}} \succ x_{j_{2}} \succ \cdots \succ x_{j_{n}}$, $y_{i_{1}} \succ y_{i_{2}} \succ \cdots \succ y_{i_{m}}$.

Step 3. Fixing initial weights of criteria satisfying median criteria components priority set in Step 2 and normalizing condition:

$$
\alpha_{j_{1}} \succ \alpha_{j_{2}} \succ \cdots \succ \alpha_{j_{n}}, \quad \beta_{i_{1}} \succ \beta_{i_{2}} \succ \cdots \succ \beta_{i_{m}}, \sum_{r=1}^{n} \alpha_{j_{r}}=\sum_{s=1}^{m} \beta_{i_{s}}=1 .
$$

Step 4. Calculation of functions $\varphi(X)$ and $\psi(Y)$ values and total value $\varphi(X)+\psi(Y)$ for each alternative.

$$
\varphi(X)=\sum_{r=1}^{n} \alpha_{j_{r}} x_{j_{r}}, \psi(Y)=\sum_{s=1}^{m} \beta_{i_{s}} y_{i_{s}} .
$$

Step 5. Calculation of ranks of alternatives according to the total value $\varphi(X)+\psi(Y): R^{(k)}(\alpha)$ and $R^{(k)}(\beta), k=1,2, \ldots, K$.

Step 6. Calculation number of elements having ranks lower than $K_{X}$ according to the criteria $X$ and lower than $K_{Y}$ according to the criteria $Y:\left|A_{K_{x}} \cap B_{K_{y}}\right|$.

Step 7. Calculation sum of squared ranks differences for the alternatives satisfying conditions of Step 6, i.e. denote $A_{K_{x}} \cap B_{K_{y}}=\left\{k \in\{1,2, \ldots, K\}: R^{(k)}(\alpha) \leqslant K_{x}, R^{(k)}(\beta) \leqslant K_{y}\right\}$. Then

$$
\sum_{k \in\left\{A_{K_{x}} \cap B_{K_{y}}\right\}}\left(R^{(k)}(\alpha)-R^{(k)}(\beta)\right)^{2} .
$$

Steps 4-7 are repeated until number of elements in Step 6 will reach its maximum value and the value of target function in Step 7 reach its minimum value. The obtained values of criteria weights $\alpha_{j_{1}}, \alpha_{j_{2}}, \cdots, \alpha_{j_{n}}, \beta_{i_{1}}, \beta_{i_{2}}, \cdots, \beta_{i_{m}}$ are used for calculation of total value $\varphi(X)+\psi(Y)$ and MCDM problem solution.

\section{Determining priority of criteria}

Say that priority of criteria $X=\left(x_{1}, x_{2}, \ldots, x_{n}\right)$ and $Y=\left(y_{1}, y_{2}, \ldots, y_{m}\right)$ was estimated by $S$ experts. Each expert sorted criteria in the priority descending order:

$$
x_{i_{1}}^{(s)} \succ x_{i_{2}}^{(s)} \succ \ldots \succ x_{i_{n}}^{(s)}, y_{i_{1}}^{(s)} \succ y_{i_{2}}^{(s)} \succ \ldots \succ y_{i_{m}}^{(s)},
$$

here $s \in\{1,2, \ldots, S\}$ - number of experts. We must ascertain generalized experts opinion. This task will be accomplished by Kemeny median method. Suppose that permutations $\left(j_{1}, j_{2}, \ldots, j_{n}\right)$ of the set of natural numbers $\{1,2, \ldots, n\}$ determine priorities of vector (criteria) $X$ components: $x_{j_{1}} \succ x_{j_{2}} \succ \cdots \succ x_{j_{n}}$. It means that for the set $\left\{x_{1}, x_{2}, \ldots, x_{n}\right\}$ a strict order relationship $R=$ $\left(x_{j_{1}}, x_{j_{2}}, \ldots, x_{j_{n}}\right)$ is defined. This relationship can be defined by the square matrix $A_{R}=\left\|a_{i j}\right\|$, 
which elements are: $a_{i j}=\left\{\begin{array}{ll}1, & \text { if } x_{i}<x_{j}, \\ 0, & \text { if } x_{i} \geqslant x_{j} .\end{array}\right.$, where $a_{i i}=0$ and $a_{i j}=1-a_{j i}$, for $i \neq j$.

Define function

$$
\rho_{A}\left(R^{(1)}, R^{(2)}\right)=\sum_{i=1}^{n} \sum_{j=1}^{n}\left|a_{i j}^{(1)}-a_{i j}^{(2)}\right| .
$$

Function (8) is a certain measure of difference between two relationships and its values coincide with values of Kemeny distance function [28].

For example, if ranks of criteria $X=\left(x_{1}, x_{2}, x_{3}, x_{4}\right)$ components are established by the first expert as $R^{(1)}=(3,2,4,1)$ and by the second expert as $R^{(2)}=(2,1,4,3)$, consequently priorities of criteria components are set accordingly $x_{4} \succ x_{2} \succ x_{1} \succ x_{3}$ and $x_{2} \succ x_{1} \succ x_{4} \succ x_{3}$. Then corresponding matrices are:

$$
A^{(1)}=\left(\begin{array}{cccc}
0 & 0 & 1 & 0 \\
1 & 0 & 1 & 0 \\
0 & 0 & 0 & 0 \\
1 & 1 & 1 & 0
\end{array}\right), A^{(2)}=\left(\begin{array}{cccc}
0 & 0 & 1 & 1 \\
1 & 0 & 1 & 1 \\
0 & 0 & 0 & 0 \\
0 & 0 & 1 & 0
\end{array}\right)
$$

Function (8) in this case is gaining value $\rho_{A}\left(R^{(1)}, R^{(2)}\right)=\sum_{i=1}^{4} \sum_{j=1}^{4}\left|a_{i j}^{(1)}-a_{i j}^{(2)}\right|=1+1+0+2=4$.

Suppose that $S$ experts established priorities $R^{(1)}, R^{(2)}, \ldots, R^{(S)}$. Most consistent with these estimates will be priority $R_{A}$, which is called median:

$$
R_{A}=\arg \min _{R} \sum_{j=1}^{S} \rho_{A}\left(R, R^{(j)}\right) .
$$

Notice that we can analyze functions $\rho_{A}$ analogues, i.e. another distances between two relationships. The solution of (9) is not necessary a unique value. Sometimes we can obtain several medians which are solutions of problem (9).

If we are interested in the majority experts opinion only (not all experts) then we apply cluster analysis procedure, that helps to distinguish a cluster of experts majority. Then the median is sought among selected group of experts.

Let's us consider an example. Priority of criteria $X=\left(x_{1}, x_{2}, x_{3}, x_{4}\right)$ and $Y=\left(y_{1}, y_{2}, y_{3}\right)$ was estimated by 5 experts. Results of estimation presented in the Table 1 . First expert set

Table 1: Criteria $X$ and $Y$ components preferences established by 5 experts.

\begin{tabular}{|c||c|c|c|c||c|c|c|}
\hline Expert & $x_{1}$ & $x_{2}$ & $x_{3}$ & $x_{4}$ & $y_{1}$ & $y_{2}$ & $y_{3}$ \\
\hline \hline 1 & 3 & 2 & 4 & 1 & 1 & 3 & 2 \\
\hline 2 & 2 & 1 & 4 & 3 & 1 & 2 & 3 \\
\hline 3 & 3 & 2 & 1 & 4 & 1 & 2 & 3 \\
\hline 4 & 2 & 3 & 4 & 1 & 2 & 1 & 3 \\
\hline 5 & 3 & 4 & 2 & 1 & 2 & 3 & 1 \\
\hline
\end{tabular}

criterion $X$ components priorities as follows: $x_{4} \succ x_{2} \succ x_{1} \succ x_{3}$ and criterion $Y$ components priorities: $y_{1} \succ y_{3} \succ y_{2}$. Notice that medians must be sought independently for criterion $X$ and $Y$ components. Let's calculate elements of the matrices $A_{X}^{(i)}$ :

$$
A_{X}^{(1)}=\left(\begin{array}{llll}
0 & 0 & 1 & 0 \\
1 & 0 & 1 & 0 \\
0 & 0 & 0 & 0 \\
1 & 1 & 1 & 0
\end{array}\right), A_{X}^{(2)}=\left(\begin{array}{llll}
0 & 0 & 1 & 1 \\
1 & 0 & 1 & 1 \\
0 & 0 & 0 & 0 \\
0 & 0 & 1 & 0
\end{array}\right), A_{X}^{(3)}=\left(\begin{array}{llll}
0 & 0 & 0 & 1 \\
1 & 0 & 0 & 1 \\
1 & 1 & 0 & 1 \\
0 & 0 & 0 & 0
\end{array}\right),
$$




$$
A_{X}^{(4)}=\left(\begin{array}{llll}
0 & 1 & 1 & 0 \\
0 & 0 & 1 & 0 \\
0 & 0 & 0 & 0 \\
1 & 1 & 1 & 0
\end{array}\right), A_{X}^{(5)}=\left(\begin{array}{cccc}
0 & 1 & 0 & 0 \\
0 & 0 & 0 & 0 \\
1 & 1 & 0 & 0 \\
1 & 1 & 1 & 0
\end{array}\right) .
$$

Note, that we must search the median $R_{A_{X}}$ among all $24=4$ ! components priorities options. Median is the priority, which minimize functions (9) value. In this case solution was found among priorities $R^{(1)}-R^{(5)}$ (for other priorities options value of function (9) is bigger):

$$
\begin{aligned}
& \sum_{j=1}^{5} \rho_{A}\left(R^{(1)}, R^{(j)}\right)=0+4+10+2+6=22, \quad \sum_{j=1}^{5} \rho_{A}\left(R^{(2)}, R^{(j)}\right)=4+0+6+6+10=26, \\
& \sum_{j=1}^{5} \rho_{A}\left(R^{(3)}, R^{(j)}\right)=10+6+0+12+8=36, \quad \sum_{j=1}^{5} \rho_{A}\left(R^{(4)}, R^{(j)}\right)=2+6+12+0+4=24, \\
& \sum_{j=1}^{5} \rho_{A}\left(R^{(5)}, R^{(j)}\right)=6+10+8+4+0=28 \quad \ldots
\end{aligned}
$$

Median components priority is $R_{A_{X}}=R^{(1)}=(3,2,4,1)$ or $x_{4} \succ x_{2} \succ x_{1} \succ x_{3}$. Next we find criterion $Y$ components priorities among all $6=3$ ! options. Calculate elements of matrices $A_{Y}^{(i)}$ :

$$
\begin{gathered}
A_{Y}^{(1)}=\left(\begin{array}{lll}
0 & 1 & 1 \\
0 & 0 & 0 \\
0 & 1 & 0
\end{array}\right), A_{Y}^{(2)}=A_{Y}^{(3)}=\left(\begin{array}{lll}
0 & 1 & 1 \\
0 & 0 & 1 \\
0 & 0 & 0
\end{array}\right), A_{Y}^{(4)}=\left(\begin{array}{lll}
0 & 0 & 1 \\
1 & 0 & 1 \\
0 & 0 & 0
\end{array}\right), \\
A_{Y}^{(5)}=\left(\begin{array}{lll}
0 & 1 & 0 \\
0 & 0 & 0 \\
1 & 1 & 0
\end{array}\right), A_{Y}^{(6)}=\left(\begin{array}{lll}
0 & 0 & 0 \\
1 & 0 & 1 \\
1 & 0 & 0
\end{array}\right), A_{Y}^{(7)}=\left(\begin{array}{lll}
0 & 0 & 0 \\
1 & 0 & 0 \\
1 & 1 & 0
\end{array}\right) .
\end{gathered}
$$

Here $R^{(6)}=(3,1,2), R^{(7)}=(3,2,1) . \sum_{j=1}^{5} \rho_{A}\left(R^{(1)}, R^{(j)}\right)=0+2+2+4+2=10$,

$\sum_{j=1}^{5} \rho_{A}\left(R^{(2)}, R^{(j)}\right)=\sum_{j=1}^{5} \rho_{A}\left(R^{(3)}, R^{(j)}\right)=2+0+0+2+4=8$,

$\sum_{j=1}^{5} \rho_{A}\left(R^{(4)}, R^{(j)}\right)=4+2+2+0+6=14, \quad \sum_{j=1}^{5} \rho_{A}\left(R^{(5)}, R^{(j)}\right)=2+4+4+6+0=16$,

$\sum_{j=1}^{5} \rho_{A}\left(R^{(6)}, R^{(j)}\right)=6+4+4+2+4=20, \quad \sum_{j=1}^{5} \rho_{A}\left(R^{(7)}, R^{(j)}\right)=4+6+6+4+2=22$.

Median components prioritiy is $R_{A_{Y}}=R^{(2)}=R^{(3)}=(1,2,3)$ or $y_{1} \succ y_{2} \succ y_{3}$.

\section{Calculation of weights and MCDM problem solution}

Suppose that there are known objective measurements $(x)$ and subjective expert evaluations (y) of $K$ test takers $T^{(j)}, j=1,2, \ldots, K: x_{1}^{(j)}, x_{2}^{(j)}, \ldots, x_{n}^{(j)}, y_{1}^{(j)}, y_{2}^{(j)}, \ldots, y_{m}^{(j)}, 0 \leqslant x_{i}^{(j)}, y_{i}^{(j)} \leqslant 1$ :

$$
T^{\left(j_{1}\right)} \succeq T^{\left(j_{2}\right)} \text {, if }(\forall i) x_{i}^{\left(j_{1}\right)} \geqslant x_{i}^{\left(j_{2}\right)} \& y_{i}^{\left(j_{1}\right)} \geqslant y_{i}^{\left(j_{2}\right)} .
$$

Suppose that $0 \leqslant w_{x i}, w_{y i} \leqslant 1$ are weighted coefficients: $\sum_{i=1}^{n} w_{x i}=\sum_{i=1}^{m} w_{y i}=1$. Then under conditions (10) the following inequalities take place:

$$
W_{x}^{\left(j_{1}\right)} \geqslant W_{x}^{\left(j_{2}\right)} \& W_{y}^{\left(j_{1}\right)} \geqslant W_{y}^{\left(j_{2}\right)}
$$

Here

$$
W_{x}^{(j)}=\sum_{i=1}^{n} w_{x i} x_{i}^{(j)}, W_{y}^{(j)}=\sum_{i=1}^{m} w_{y i} y_{i}^{(j)}
$$


It means that if all measurements values are bigger for object $T^{\left(j_{1}\right)}$ than for object $T^{\left(j_{2}\right)}$ then each linear combination of measurements for $T^{\left(j_{1}\right)}$ will also be bigger than for $T^{\left(j_{2}\right)}$ provided that weighted coefficients are nonnegative. In practice more often we have encountering situation when results of the measurements are as follows: $x_{i_{1}}^{\left(j_{1}\right)}>x_{i_{1}}^{\left(j_{2}\right)}$ and $x_{i_{2}}^{\left(j_{1}\right)}<x_{i_{2}}^{\left(j_{2}\right)}$. Then it isn't possible to apply criteria (10) for selecting better alternative. The idea of current study is to choose such values of weights $w_{x i}, w_{y i}$ which will guarantee proximity of values $W_{x}^{(j)}$ and $W_{y}^{(j)}$ for $10 \%$ of best security guards. The measure of closeness of these values would be sum of squares of ranks differences.

Let's denote $R_{x}^{(j)}$ and $R_{y}^{(j)}$ positive integers $R_{x, y}^{(j)} \in\{1,2, \ldots, K\}$ satisfying condition: $R_{x, y}^{\left(j_{1}\right)}<$ $R_{x, y}^{\left(j_{2}\right)}$, when $W_{x, y}^{\left(j_{1}\right)}>W_{x, y}^{\left(j_{2}\right)}$, i.e. they are ranks of numbers $W_{x, y}^{(j)}$. Let's define $A_{K_{x}}$ and $B_{K_{y}}$ as subsets of the set $\{1,2, \ldots, K\}: A_{K_{x}}=\left\{\left\{j_{1}, j_{2}, \ldots, j_{K_{x}}\right\}: R_{x}^{\left(j_{i}\right)} \leqslant K_{x}\right\}$,

$B_{K_{y}}=\left\{\left\{j_{1}, j_{2}, \ldots, j_{K_{y}}\right\}: R_{y}^{\left(j_{i}\right)} \leqslant K_{y}\right\}$ including objects which have lowest ranks according to the corresponding criteria ( $X$ and $Y$ ) and having $K_{x}$ and $K_{y}$ elements respectively. Note, that the lower is the rank, the better is the alternative.

The best alternatives selection task according to both criteria (12) is equivalent to the task of finding the intersection of sets $A_{K_{x}} \cap B_{K_{y}}$, which has the required number of elements $\left|A_{K_{x}} \cap B_{K_{y}}\right|$. If the number of elements $\left|A_{K_{x}} \cap B_{K_{y}}\right|$ is insufficient, it is necessary to increase numbers $K_{x}$ and $K_{y}$. Preferences of the selected alternatives could be determined by the following criteria expressed as sum of criteria $(12): W^{(j)}=W_{x}^{(j)}+W_{y}^{(j)}$.

Consider the following example. Suppose that 5 objects have 4 attributes of one type $(X)$ and 3 attributes of other type $-(Y)$. Values of attributes are given in the Table 2 . Let's calculate

Table 2: Attributes $x_{i}$ and $y_{j}$ values for the 5 given objects.

\begin{tabular}{|c||c|c|c|c||c|c|c|}
\hline & $x_{1}$ & $x_{2}$ & $x_{3}$ & $x_{4}$ & $y_{1}$ & $y_{2}$ & $y_{3}$ \\
\hline \hline 1 & 0.4 & 0.6 & 0.7 & 0.3 & 0.5 & 0.4 & 0.2 \\
\hline 2 & 0.5 & 0.3 & 0.7 & 0.3 & 0.6 & 0.7 & 0.2 \\
\hline 3 & 0.2 & 0.4 & 0.6 & 0.5 & 0.7 & 0.7 & 0.4 \\
\hline 4 & 0.5 & 0.3 & 0.5 & 0.4 & 0.6 & 0.8 & 0.2 \\
\hline 5 & 0.6 & 0.2 & 0.8 & 0.8 & 0.4 & 0.5 & 0.8 \\
\hline
\end{tabular}

values of criteria (12) for the first object when weights are as follows:

$w_{x, 1}=\frac{1}{2}, w_{x, 2}=\frac{1}{4}, w_{x, 3}=\frac{1}{8}, w_{x, 4}=\frac{1}{8}, w_{y, 1}=\frac{5}{8}, w_{y, 2}=\frac{1}{4}, w_{y, 3}=\frac{1}{8}$. We obtain criteria (12) values: $W_{x}^{(1)}=\frac{1}{2} 0.4+\frac{1}{4} 0.6+\frac{1}{8} 0.7+\frac{1}{8} 0.3=0.475, W_{y}^{(1)}=\frac{5}{8} 0.5+\frac{1}{4} 0.4+\frac{1}{8} 0.2=0.4375$.

Similarly calculate criteria values for the remaining 4 objects. All criteria values and their ranks are presented in the Table 3. Previously defined sets $A_{K_{x}}$ and $B_{K_{y}}$ are those including

Table 3: Criteria $W_{x}^{(j)}$ and $W_{y}^{(j)}$ and their ranks values for the 5 given objects.

\begin{tabular}{|c||c|c|c|c|}
\hline$j$ & $W_{x}^{(j)}$ & $W_{y}^{(j)}$ & $R_{x}^{(j)}$ & $R_{y}^{(j)}$ \\
\hline \hline 1 & 0.475 & 0.4375 & 2 & 5 \\
\hline 2 & 0.45 & 0.575 & 3 & 3 \\
\hline 3 & 0.3375 & 0.6625 & 5 & 1 \\
\hline 4 & 0.4375 & 0.60 & 4 & 2 \\
\hline 5 & 0.55 & 0.475 & 1 & 4 \\
\hline
\end{tabular}

respectively $K_{x}$ and $K_{y}$ elements with lowest ranks $R_{x}^{(j)}$ and $R_{y}^{(j)}: A_{1}=\{5\}, A_{2}=\{1,5\}$, $A_{3}=\{1,2,5\}, A_{4}=\{1,2,4,5\}, B_{1}=\{3\}, B_{2}=\{3,4\}, B_{3}=\{2,3,4\}, B_{4}=\{2,3,4,5\}$, $A_{1} \cap B_{1}=\varnothing, A_{2} \cap B_{2}=\varnothing, A_{3} \cap B_{3}=\{2\}$ and so on. 
Calculation of criteria weights is performed simultaneously with the decision of MCDM problem. Suppose that there are known criteria-referenced assessments for certain alternatives $X^{(k)}=\left(x_{1}^{(k)}, x_{2}^{(k)}, \ldots, x_{n}^{(k)}\right), Y^{(k)}=\left(y_{1}^{(k)}, y_{2}^{(k)}, \ldots, y_{m}^{(k)}\right), k=1,2, \ldots, K$ and criteria $X, Y$ priorities are determined:

$$
x_{j_{1}} \succ x_{j_{2}} \succ \cdots \succ x_{j_{n}}, \quad y_{i_{1}} \succ y_{i_{2}} \succ \cdots \succ y_{i_{m}}
$$

According to the established criteria $X, Y$ priorities (13) functions $\varphi(X)$ and $\psi(Y)$ are determined as follows:

$$
\begin{gathered}
\varphi(X)=\sum_{r=1}^{n} \alpha_{j_{r}} x_{j_{r}}, \text { where } \alpha_{j_{1}} \geqslant \alpha_{j_{2}} \geqslant \ldots \geqslant \alpha_{j_{n}}>0 \\
\psi(Y)=\sum_{s=1}^{m} \beta_{i_{s}} y_{i_{s}}, \text { where } \beta_{i_{1}} \geqslant \beta_{i_{2}} \geqslant \ldots \geqslant \beta_{i_{m}}>0 .
\end{gathered}
$$

For identifying criteria weights we use the heuristic described in [13]. Require that the weighting coefficients $\alpha_{j}$ and $\beta_{i}$ satisfy normalizing condition (4).

Denote $\mathcal{S}_{\left(j_{1}, j_{2}, \ldots, j_{n}\right)}$ class of all convolutions constructed on the base of weighted averages and having priority feature $\left(j_{1}, j_{2}, \ldots, j_{n}\right)$, i.e. satisfying condition $(14)$. Analogous $\mathcal{S}_{\left(i_{1}, i_{2}, \ldots, i_{m}\right)}$ is a class of all convolutions having priority feature $\left(i_{1}, i_{2}, \ldots, i_{m}\right)$ (satisfying condition $\left.(15)\right)$.

Suppose that $\varphi$ and $\psi$ are criteria $X$ and $Y$ convolutions having corresponding priority features (13). For each alternative $\left(X^{(k)}, Y^{(k)}\right)$ we'll calculate values of both criteria convolutions $\varphi\left(X^{(k)}\right), \psi\left(Y^{(k)}\right)$. Numbering them in ascending order we get ranks of alternatives: $R_{x}^{(k)}, R_{y}^{(k)}$.

Denote $A_{K_{x}}$ and $B_{K_{y}}$ sets of the best alternatives - i. e. those subsets of the set $\{1,2, \ldots, K\}$, whose elements ranks satisfy the inequalities $R_{x}^{(k)} \leqslant K_{x}$ and $R_{y}^{(k)} \leqslant K_{y}$. In the set $A_{K_{x}}$ there are $K_{x}$ the best alternatives according to criteria $X$, similarly in the set $B_{K_{y}}$ there are $K_{y}$ the best alternatives according to criteria $Y$. Numbers $K_{x}$ ir $K_{y}$ are chosen so that the intersection of sets $A_{K_{x}} \cap B_{K_{y}}$ have not less than $10 \%$ of the best alternatives.

Then we search such functions $\varphi$ and $\psi$ that the number of elements of sets $A_{K_{x}}$ and $B_{K_{y}}$ intersection should be the maximum:

$$
\begin{aligned}
& \max _{\varphi \in \mathcal{S}_{\left(j_{1}, j_{2}, \ldots, j_{n}\right)}}\left|A_{K_{x}} \cap B_{K_{y}}\right| \\
& \psi \in \mathcal{S}_{\left(i_{1}, i_{2}, \ldots, i_{m}\right)}
\end{aligned}
$$

Condition (16) means that we must select convolutions $\varphi$ and $\psi$, which maximize criteria $X$ and $Y$ compatibility. The number of convolutions $\varphi$ and $\psi$, maximizing (16) can be great, therefore additional optimization problem must be formulated.

Denote $R^{(k)}(\alpha)$ and $R^{(k)}(\beta)$ ranks of the numbers $\left\{\varphi\left(X^{(1)}\right), \varphi\left(X^{(2)}\right), \ldots, \varphi\left(X^{(K)}\right)\right\}$ and $\left\{\psi\left(Y^{(1)}\right), \psi\left(Y^{(2)}\right), \ldots, \psi\left(Y^{(K)}\right)\right\}$ respectively $(k=1,2, \ldots, K)$.

We'll apply Indicator Rank Accordance method for minimizing ranks discrepancy function, i. e. sum of squares of the highest ranks differences according to criteria $X$ and $Y$ :

$$
C R_{K_{x}, K_{y}}(\alpha, \beta)=\min _{\substack{\varphi \in \mathcal{S}_{\left(j_{1}, j_{2}, \ldots, j_{n}\right)} \\ \psi \in \mathcal{S}_{\left(i_{1}, i_{2}, \ldots, i_{m}\right)}}} \sum_{k \in\left\{A_{K_{x}} \cap B_{K_{y}}\right\}}\left(R^{(k)}(\alpha)-R^{(k)}(\beta)\right)^{2} .
$$

Here $K_{x}$ and $K_{y}$ are chosen so that the number of elements in the intersection $A_{K_{x}} \cap B_{K_{y}}$ will be equal to the desired number of selected objects. 
For example, suppose that for the data given in Table 2 five experts proposed criteria priorities presented in Table 1 . In Chapter 3 for these data we have determined criteria priority features as follows: $x_{4} \succ x_{2} \succ x_{1} \succ x_{3}, \quad y_{1} \succ y_{2} \succ y_{3}$.

Therefore, we'll search for weights values, satisfying conditions

$$
\alpha_{4} \geqslant \alpha_{2} \geqslant \alpha_{1} \geqslant \alpha_{3}, \quad \beta_{1} \geqslant \beta_{2} \geqslant \beta_{3}
$$

and normalizing conditions (4) which maximize number of elements in the intersection of sets (16) and minimize value of function (17). Let's choose $K_{x}=K_{y}=3$ and initial values of coefficients as follows: $\alpha_{1}=\frac{1}{12}, \alpha_{2}=\frac{1}{3}, \alpha_{3}=\frac{1}{12}, \alpha_{4}=\frac{1}{2}, \beta_{1}=\beta_{2}=\beta_{3}=\frac{1}{3}$. Values of functions $\varphi\left(X^{(i)}\right)$ and $\psi\left(Y^{(i)}\right)$ and their ranks for each alternative are given in Table 4.

Table 4: Values of functions $\varphi\left(X^{(i)}\right)$ and $\psi\left(Y^{(i)}\right)$ and their ranks values for the 5 given objects.

\begin{tabular}{|c||c|c|c|c|}
\hline$j$ & $W_{x}^{(j)}$ & $W_{y}^{(j)}$ & $R_{x}^{(j)}$ & $R_{y}^{(j)}$ \\
\hline \hline 1 & 0.4417 & 0.33 & 3 & 5 \\
\hline 2 & 0.35 & 0.45 & 5 & 4 \\
\hline 3 & 0.45 & 0.54 & 2 & 1 \\
\hline 4 & 0.3833 & 0.48 & 4 & 3 \\
\hline 5 & 0.5833 & 0.51 & 1 & 2 \\
\hline
\end{tabular}

$A_{3}=\{1,3,5\}, B_{3}=\{3,4,5\}, A_{3} \cap B_{3}=\{3,5\},\left|A_{3} \cap B_{3}\right|=2$.

So, in the sum (17) we have only 2 summands: $C R_{3,3}(\alpha, \beta)=(2-1)^{2}+(1-2)^{2}=2$.

In the next step we choose other values of coefficients satisfying conditions (18) and (4). If the number of elements in the intersection of sets (16) is greater or equal than 2, we calculate function (17) value. If this value is less than in previous step, values of these new coefficients are considered to be the best solution before the next step. After the last step when all coefficients satisfying conditions (18) and (4) are verified we'll receive values of coefficients $\alpha_{1}, \alpha_{2}, \alpha_{3}, \alpha_{4}, \beta_{1}, \beta_{2}, \beta_{3}$ which are the solution of MCDM problem. Finally, values of functions $\varphi(X)$ and $\psi(Y)$ are calculated and objects are ranked according to the criteria $\varphi(X)+\psi(Y)$.

\section{Case study}

The present problem has been solved in [13]. In the mentioned paper weighted coefficients of criteria were calculated as the proportions of the collected scores to the total score. In the current research the weights obtained by KEMIRA method. Results of selecting the best $10 \%$ security guards will be compared with previous results. Moreover, guard ranking will be performed.

118 security guards were randomly selected from the company G4S Lietuva. 22 leader managers (experts) ranked the competences described below.

Personnel elite $-10 \%$ the best employees of the private security company - are selected according to six internal assessment criteria $\left(x_{1}-x_{6}\right)$ and nine external evaluation criteria $\left(y_{1}-y_{9}\right)$. 6 internal criteria are objective tests and measurements: $x_{1}$ is employee's theoretical and practical preparation; $x_{2}$ - professional activity, $x_{3}$ - mental qualities; $x_{4}$ - physical development; $x_{5}$ - motor abilities (personal physical conditions allowing to carry out physical tasks); $x_{6}$ - fighting efficiency. 9 external criteria are evaluation of subordinate by his immediate superior: $y_{1}-$ specialty knowledge, professionalism, $y_{2}$ - diligence and positive attitude to work, $y_{3}$ - behavior with colleagues and supervisors; $y_{4}$ - reliability at work; $y_{5}$ - quality of work; $y_{6}$ - workload performance; $y_{7}$ - image; $y_{8}$ - development rate; $y_{9}$ - being promising (potential to make a career). Fragment of security guards evaluation criteria structure is given in Table $5 . \quad$ Table 5 data are given in the paper [13]. Note that all 15 criteria are associated with benefit and their 
Table 5: Security guards internal and external evaluation criteria.

\begin{tabular}{|c||c|c|c|c||c|c|c|c|}
\hline \multirow{2}{*}{ Security guards } & \multicolumn{3}{c|}{ Internal criteria } & \multicolumn{3}{c|}{ External criteria } \\
\cline { 2 - 9 } & $x_{1}$ & $x_{2}$ & $\ldots$ & $x_{6}$ & $y_{1}$ & $y_{2}$ & $\ldots$ & $y_{9}$ \\
\hline$a_{1}$ & $\ldots$ & $\ldots$ & $\ldots$ & $\ldots$ & $\ldots$ & $\ldots$ & $\ldots$ & $\ldots$ \\
\hline$\ldots$ & $\ldots$ & $\ldots$ & $\ldots$ & $\ldots$ & $\ldots$ & $\ldots$ & $\ldots$ & $\ldots$ \\
\hline$a_{118}$ & $\ldots$ & $\ldots$ & $\ldots$ & $\ldots$ & $\ldots$ & $\ldots$ & $\ldots$ & $\ldots$ \\
\hline
\end{tabular}

greater value is better. Therefore criteria values were transformed to values $x_{i}^{j *}, y_{i}^{j *}$, belonging to the range $[0 ; 1]$ by the transformations $(2) .22$ independent experts determined preferences separately for internal and external evaluation criteria. Criteria preferences established by experts are presented in the Table 6. A higher grade means that the criterion is more important.

Criterion $X$ priority feature is determined from expert estimates of the form $\left(j_{1}, j_{2}, \ldots, j_{6}\right)$. For example, first expert set criterion $X$ components priorities as follows: $\left(x_{1}, x_{3}, x_{6}, x_{4}, x_{5}, x_{2}\right)$. Here $x_{1}$ is the most important and $x_{2}$ - the least important component. Criterion $Y$ priority feature is established similarly. For example, first expert set them in this order: $\left(y_{2}, y_{4}, y_{1}, y_{7}, y_{5}, y_{3}\right.$, $\left.y_{6}, y_{8}, y_{9}\right)$. Generalized experts opinion expressing criteria priority feature is established by calculating median with the respective metric (8) to calculate distances between priorities. Calculation of medians was performed by full re-selection of options, which is respectively $6 !=720$ and $9 !=362880$.

Medians were calculated separately for criterion $X$ components $x_{j}$ and criterion $Y$ components $y_{i}$ by minimizing function (9) values. Two solutions were obtained for criterion $X$ components $x_{j}$ priority features: $(1,5,3,6,4,2),(1,3,5,6,4,2)$. Criterion $Y$ components $y_{i}$ priority features were determined uniquely: $(2,4,5,3,1,7,6,8,9)$. Therefore, criteria $X$ and $Y$ components order was established respectively:

$$
\begin{gathered}
x_{1} \succ x_{5} \succ x_{3} \succ x_{6} \succ x_{4} \succ x_{2} \quad \text { or } \quad x_{1} \succ x_{3} \succ x_{5} \succ x_{6} \succ x_{4} \succ x_{2}, \\
y_{2} \succ y_{4} \succ y_{5} \succ y_{3} \succ y_{1} \succ y_{7} \succ y_{6} \succ y_{8} \succ y_{9} .
\end{gathered}
$$

Table 6: Criteria $X$ and $Y$ components preferences established by experts.

\begin{tabular}{|c|c|c|}
\hline Expert & $x_{1} \cdots x_{6}$ & $y_{1} \cdots y_{9}$ \\
\hline 1 & 615324 & 794853621 \\
2 & 523164 & 795682341 \\
3 & 513264 & 594681732 \\
4 & 615342 & 597863421 \\
5 & 615234 & 796853421 \\
6 & 514263 & 596873421 \\
7 & 423165 & 485972631 \\
8 & 625341 & 685973421 \\
9 & 546321 & 794863512 \\
10 & 546123 & 674983521 \\
11 & 413265 & 586972431 \\
\hline
\end{tabular}

\begin{tabular}{|c|c|c|}
\hline Expert & $x_{1} \cdots x_{6}$ & $y_{1} \cdots y_{9}$ \\
\hline 12 & 624153 & 695784321 \\
13 & 645132 & 596873421 \\
14 & 635142 & 786954321 \\
15 & 413265 & 597863241 \\
16 & 413265 & 798654231 \\
17 & 614235 & 687945231 \\
18 & 634152 & 698745231 \\
19 & 415263 & 498671532 \\
20 & 624153 & 596783421 \\
21 & 526341 & 687954321 \\
22 & 416325 & 697854231 \\
\hline
\end{tabular}

Finally, cluster analysis [17] was applied to the data presented in the Table 6 to distinguish a group of experts whose opinions are very close to each other and which form the majority of the experts. By applying Between groups, Ward's and Furthest neighbor methods the group of experts with numbers $\{1,4,5,8,9,10,12,13,14,17,18,20,21,22\}$ was selected. Further only those experts priorities were examined to get criteria priority preferences, because we did not want to distort the opinion of this group by the remaining minority group of experts. Kemeny median method was applied for this reduced data array. For criterion $X$ components $x_{j}$ the solution 
minimizing function (9) received as follows: $(1,3,5,6,2,4)$. Results for criterion $Y$ components $y_{j}$ were the same as in (20). Therefore, for the majority of experts criteria $X$ and $Y$ components order was established respectively:

$$
x_{1} \succ x_{3} \succ x_{5} \succ x_{6} \succ x_{2} \succ x_{4}
$$

and (20). From the priority features (19), (21) and (20) it follows, that it is necessary to search for convolutions $\varphi$ and $\psi$ in the form:

$$
\begin{gathered}
\varphi_{1}(X)=\alpha_{1} x_{1}+\alpha_{5} x_{5}+\alpha_{3} x_{3}+\alpha_{6} x_{6}+\alpha_{4} x_{4}+\alpha_{2} x_{2}, \quad \alpha_{1} \geqslant \alpha_{5} \geqslant \alpha_{3} \geqslant \alpha_{6} \geqslant \alpha_{4} \geqslant \alpha_{2}>0, \\
\varphi_{2}(X)=\alpha_{1} x_{1}+\alpha_{3} x_{3}+\alpha_{5} x_{5}+\alpha_{6} x_{6}+\alpha_{4} x_{4}+\alpha_{2} x_{2}, \quad \alpha_{1} \geqslant \alpha_{3} \geqslant \alpha_{5} \geqslant \alpha_{6} \geqslant \alpha_{4} \geqslant \alpha_{2}>0, \\
\varphi_{3}(X)=\alpha_{1} x_{1}+\alpha_{3} x_{3}+\alpha_{5} x_{5}+\alpha_{6} x_{6}+\alpha_{2} x_{2}+\alpha_{4} x_{4}, \quad \alpha_{1} \geqslant \alpha_{3} \geqslant \alpha_{5} \geqslant \alpha_{6} \geqslant \alpha_{2} \geqslant \alpha_{4}>0, \\
\psi(Y)=\beta_{2} y_{2}+\beta_{4} y_{4}+\cdots+\beta_{9} y_{9}, \quad \beta_{2} \geqslant \beta_{4} \geqslant \beta_{5} \geqslant \cdots \geqslant \beta_{8} \geqslant \beta_{9}>0 .
\end{gathered}
$$

We define parameter values for our problem: number of investigated objects $K=118$, number of criterion $X$ components $n=6$, number of criterion $Y$ components $m=9$. Values $K_{x}=21$ and $K_{y}=21$ were chosen to fulfill the condition $\left|A_{K_{x}} \cap B_{K_{y}}\right|=12$, since the goal is to select the top 12 security guards. In the case of functions $\varphi_{1}(X)$ and $\psi(Y)$ the lowest criterion (17) value was found $C R_{21,21}(\alpha, \beta)=331$ with the following values of weighted coefficients:

\begin{tabular}{|c|c|c|c|c|c|c|c|c|c|c|c|c|c|c|}
\hline$\alpha_{1}$ & $\alpha_{5}$ & $\alpha_{3}$ & $\alpha_{6}$ & $\alpha_{4}$ & $\alpha_{2}$ & $\beta_{2}$ & $\beta_{4}$ & $\beta_{5}$ & $\beta_{3}$ & $\beta_{1}$ & $\beta_{7}$ & $\beta_{6}$ & $\beta_{8}$ & $\beta_{9}$ \\
\hline 0.202 & 0.202 & 0.197 & 0.191 & 0.11 & 0.098 & 0.32 & 0.149 & 0.14 & 0.12 & 0.085 & 0.064 & 0.053 & 0.035 & 0.034 \\
\hline
\end{tabular}

The best 12 security guards, belonging to intersection of sets $A_{21} \cap B_{21}$ and ranked according to the criterion $W_{1}^{(j)}=\varphi_{1}\left(x^{(j)}\right)+\psi\left(y^{(j)}\right)$ as follows: $a_{56} \succ a_{76} \succ a_{91} \succ a_{81} \succ a_{21} \succ$ $a_{47} \succ a_{36} \succ a_{106} \succ a_{34} \succ a_{111} \succ a_{102} \succ a_{77}$. In the case of functions $\varphi_{2}(X)$ and $\psi(Y)$ the lowest criterion (17) value $C R_{21,21}(\alpha, \beta)=362$ was obtained with these weighted coefficients values:

\begin{tabular}{|c|c|c|c|c|c|c|c|c|c|c|c|c|c|c|}
\hline$\alpha_{1}$ & $\alpha_{3}$ & $\alpha_{5}$ & $\alpha_{6}$ & $\alpha_{4}$ & $\alpha_{2}$ & $\beta_{2}$ & $\beta_{4}$ & $\beta_{5}$ & $\beta_{3}$ & $\beta_{1}$ & $\beta_{7}$ & $\beta_{6}$ & $\beta_{8}$ & $\beta_{9}$ \\
\hline 0.194 & 0.192 & 0.188 & 0.182 & 0.132 & 0.11 & 0.29 & 0.24 & 0.094 & 0.089 & 0.082 & 0.078 & 0.055 & 0.055 & 0.017 \\
\hline
\end{tabular}

The same 12 security guards got to the intersection of sets $A_{21} \cap B_{21}$. Alternatives ranked according to the criterion $W_{2}^{(j)}=\varphi_{2}\left(x^{(j)}\right)+\psi\left(y^{(j)}\right)$ as follows: $a_{56} \succ a_{76} \succ a_{91} \succ a_{81} \succ a_{21} \succ$ $a_{47} \succ a_{36} \succ a_{106} \succ a_{111} \succ a_{34} \succ a_{102} \succ a_{77}$. Two alternatives have exchanged places: $a_{111}$ and $a_{34}$. The obtained results coincide with the results got in [13], since in the case of $\varphi_{2}(X)$ and $\psi(Y)$ criteria priorities were set in the same order as in the mentioned article. For the generalized opinion of the experts majority determined by cluster analysis the solution was sought between the functions of the form $\varphi_{3}(X)$ and $\psi(Y)$. The minimum (17) value $C R_{19,19}(\alpha, \beta)=373$ was obtained with these weighted coefficients values:

\begin{tabular}{|c|c|c|c|c|c|c|c|c|c|c|c|c|c|c|}
\hline$\alpha_{1}$ & $\alpha_{3}$ & $\alpha_{5}$ & $\alpha_{6}$ & $\alpha_{2}$ & $\alpha_{4}$ & $\beta_{2}$ & $\beta_{4}$ & $\beta_{5}$ & $\beta_{3}$ & $\beta_{1}$ & $\beta_{7}$ & $\beta_{6}$ & $\beta_{8}$ & $\beta_{9}$ \\
\hline 0.2 & 0.19 & 0.19 & 0.19 & 0.133 & 0.097 & 0.25 & 0.249 & 0.12 & 0.1 & 0.081 & 0.07 & 0.05 & 0.05 & 0.03 \\
\hline
\end{tabular}

The top 12 security guards are: $a_{21}, a_{34}, a_{36}, a_{47}, a_{56}, a_{72}, a_{76}, a_{81}, a_{91}, a_{102}, a_{106}, a_{111}$. The difference from the previous cases when solutions were sought amongst functions $\varphi_{1}(X)$ or $\varphi_{2}(X)$ is that $a_{77}$ was changed by $a_{72}$. Alternatives ranked according to the criterion $W_{3}^{(j)}=\varphi_{3}\left(x^{(j)}\right)+$ $\psi\left(y^{(j)}\right)$ as follows: $a_{56} \succ a_{91} \succ a_{76} \succ a_{81} \succ a_{21} \succ a_{36} \succ a_{111} \succ a_{47} \succ a_{106} \succ a_{34} \succ a_{102} \succ a_{72}$. Ranking results differ from those accomplished by criteria $W_{1}^{(j)}$ and $W_{2}^{(j)}$ much more. 


\section{Discussion and conclusions}

Many authors studied the relationship between the set of qualitative indicators of human resources and business performance. Recently, this phenomenon is gaining considerable economic importance. A lot of recruitment methods, whose main aim is to help organizations make the best personnel management decisions, are being created. Many authors have expressed concern about the human resources performance assessment process because it can be biased due to the non-compliance of methods [1]. Traditional human resource evaluation and selection methods are usually based on a statistical analysis for evaluation of objective indicators thought to reflect the realities. However, it is important to assess not only known but also unknown, hard-diagnosable factors. Solution of this problem requires modern techniques involving large amounts of uncertain and subjective information [38]. For the assessment of indicators it is necessary to apply a taxonomy based sorting principles [7]. The different types of equally important factors should be grouped into separate sub-criteria [41]. There can be two or more sub-criteria. In selection process sub-criteria are given the same weights. This paper presents an algorithm which opens possibilities for recruitment in compliance with the above requirements. Sub-criteria applied to the evaluation of candidates reflect subjective and objective information about the candidates. In the current paper two equally important hierarchical structures were generated and a new method for ranking proposed. This method combines expert (subjective) evaluation and testing indicators (objective evaluation) hierarchical layout.

In the article a new approach to MCDM problem, when objects are evaluated by two groups of criteria having different origin, is presented. In the first stage criteria components preferences are established separately in each group by applying the novel method of Kemeny medians. Method of Kemeny medians can be used when information about criteria components preferences established by independent experts is available. In the second stage criteria weights are determined and MCDM problem is solved by applying proposed Indicator Rank Accordance method. Principle of this method is to choose criteria weights values from the set of all possible values which maximize number of elements having lowest ranks according to the both criteria (16) and minimize sum of squared ranks differences calculated for two groups of criteria (17). A case study of selecting top 12 security guards was analyzed by KEMIRA method. For the proposed data method of Kemeny medians was applied twice: for all experts and for the majority group of experts distinguished by cluster analysis methods.

The proposed methodology allows to weigh and synthesize subjective (managers assessments) and objective (the candidates skills) indicators. In general, there can be more than two groups of criteria having different origin. Method of Kemeny medians is useful for a wide range of MCDM problems when priority of criteria must be established according to experts evaluation. New KEMIRA method opens up the new opportunities of application and development of decisionmaking methods not only in the selection of personnel. It is suggested that this type of research could be extended to other areas of human activities where MCDM problems arise (business, manufacturing, trade and etc).

\section{Bibliography}

[1] A. Afshari, M. Mojahed, R.M. Yusuff (2010); Simple additive weighting approach to personnel selection problem, International Journal of Innovation, Management and Technology, 1(5): $511-515$.

[2] P. Arezes, M. Neves, S. Teixeira, C. Leão, J. Cunha (2013);Testing thermal comfort of trekking boots: An objective and subjective evaluation. Applied Ergonomics, 44(4): 557- 
565.

[3] A. Arisha, W. Abo-Hamad (2013); Towards Operations Excellence: Optimising Staff Scheduling For New Emergency Department. Proceedings of the 20th International Annual EurOMA Conference - "Operations Management at the Heart of the Recovery", 9-12 June 2013, (pp. 1-10). Dublin, Ireland.

[4] A. Baležentis, T. Baležentis, W.K.M. Brauers (2012); Personnel selection based on computing with words and fuzzy MULTIMOORA. Expert Systems with Applications, 39(9): 7961-7967.

[5] A. Baležentis, T. Baležentis, W.K.M. Brauers (2012); MULTIMOORA-FG: a multi-objective decision making method for linguistic reasoning with an application to personnel selection. Informatica, 23(2): 173-190.

[6] S. Balli, S. Korukoğlu (2014); Development of a fuzzy decision support framework for complex multi-attribute decision problems: a case study for the selection of skilful basketball players. Expert Systems, 31(1): 56-69.

[7] J. Bednarik, W. Andreff, S. Popović, D. Jakšić, E. Kolar, G. Jurak (2013); Financial taxonomy of non-governmental sports organizations, Kinesiology, 45(2): 241-251.

[8] J. van den Bergh, J. Belien, P. De Bruecker, E. Demeulemeester, L. De Boeck (2013); Personnel scheduling: A literature review. European Journal of Operational Research, 226(3): $367-385$.

[9] C. Chen, P. Pai, W. Hung (2011); Applying linguistic VIKOR and knowledge map in personnel selection. Asia Pacific Management Review, 16(4): 491-502.

[10] L.-C. Cheng, H.-A. Wang (2014); A fuzzy recommender system based on the integration of subjective preferences and objective information, Applied Soft Computing, 18: 290-301.

[11] S. Dadeło (2005); Czynniki determinujące kompetencje pracowników ochrony na Litwie. AWF Warszawa-Vilnius. [in Polish, abstract in English, in Lithuanian]

[12] S. Dadelo, Z. Turskis, E.K. Zavadskas, R. Dadelienè, (2013);Integrated multi-criteria decision making model based on wisdom-of-crowds principle for selection of the group of elite security guards, Archives of Budo, 9(2): 135-147.

[13] S. Dadelo, A. Krylovas, N. Kosareva, E.K. Zavadskas, R. Dadelienè (2014); Algorithm of maximizing the set of common solutions for several MCDM problems and it's application for security personnel scheduling, International journal of computers, communications $\&$ Control, 9(2): 140-148.

[14] M. Dağdeviren (2010); A hybrid multi-criteria decision-making model for personnel selection in manufacturing systems, Journal of Intelligent Manufacturing, 21(4): 451-460.

[15] A. Dahlstrom, M. Campbell, C. Hewitt (2013); The role of uncertainty and subjective influences on consequence assessment by aquatic biosecurity experts. Journal of Environmental Management, 127(30): 103-113.

[16] M. El-Santawy, R. El-Dean (2012); On using VIKOR for ranking personnel problem. Life Science Journal-Acta, 9(4): 1534-1536.

[17] V. Estivill-Castro (2002); Why so many clustering algorithms - A Position Paper. ACM SIGKDD Explorations Newsletter 4(1): 65-75. 
[18] J. Fadyl, K. Mcpherson, P. Schlüter, L. Turner-Stokes (2010); Factors contributing to workability for injured workers: literature review and comparison with available measures. Disability and Rehabilitation, 32(14): 1173-1183.

[19] S. Fazli, H. Jafari (2002); Developing a hybrid multi-criteria model for investment in stock exchange. Management Science Letters, 2(2): 457-468, 2002.

[20] B. Garland B, N. Hogan, T. Kelley, B. Kim, E. Lambert E. (2013); To Be or Not to Be Committed: The Effects of Continuance and Affective Commitment on Absenteeism and Turnover Intent among Private Prison Personnel. Journal of Applied Security Research, 8(1): $1-23$.

[21] A. Gasiorowska (2014); The relationship between objective and subjective wealth is moderated by financial control and mediated by money anxiety, Journal of Economic Psychology, 43: $64-74$.

[22] J. Grand, J. Golubovich, A. Ryan, N. Schmitt (2013); The detection and influence of problematic item content in ability tests: An examination of sensitivity review practices for personnel selection test development. Organizational behavior and human decision processes, 121(2): 158-173.

[23] S. Hashemkhani Zolfani, J. Antuchevičienè (2012); Team member selecting based on AHP and TOPSIS grey. Inzinerine Ekonomika - Engineering Economics, 23(4): 425-434.

[24] S. Hashemkhani Zolfani, N. Rezaeiniya, M. Aghdaie, E.K. Zavadskas (2012); Quality control manager selection based on AHP-COPRAS-G method: a case in Iran. Ekonomska istraživanja - Economic Research, 25(1): 88-104.

[25] M. Kabak (2013); A fuzzy DEMATEL-ANP based multi criteria decision making approach for personnel selection. Journal of Multiple-Valued Logic and Soft Computing, 20(5-6): 571593.

[26] M. Kabak, S. Burmaoğlu, Y. Kazançoğlu (2012); A fuzzy hybrid MCDM approach for professional selection. Expert Systems with Applications, 39(3): 3516-3525.

[27] A. Kelemenis, D. Askounis, A new TOPSIS-based multi-criteria approach to personnel selection. Expert Systems with Applications, 37(7): 4999-5008, 2010.

[28] J. Kemeny, J. Snell (1963); Mathematical Models in the Social Sciences, New York.

[29] M. Kendall (1970); Rank correlation methods, fourth ed., Charles Griffin \& Co., London.

[30] V. Keršulienė, Z. Turskis (2013); An Integrated Multi-criteria Group Decision Making Process: Selection of the Chief Accountant. The 2-nd International Scientific conference "Contemporary Issues in Business, Management and Education 2013", Procedia - Social and Behavioral Sciences, 110: 897-904.

[31] G. Kou, Y. Lu, Y. Peng, Y. Shi (2012); Evaluation of classification algorithms using MCDM and rank correlation. International Journal of Information Technology $\& 5$ Decision Making, 11(1): 197-225.

[32] A. Krylovas, E.K. Zavadskas, N. Kosareva, S. Dadelo (2014); New KEMIRA method for determining criteria priority and weights in solving MCDM problem. International Journal of Information Technology \& Decision Making, 13(6): 1119-1133. 
[33] J. Liou, G. Tzeng (2012); Comments on "Multiple criteria decision making (MCDM) methods in economics: an overview". Technological and Economic Development of Economy, 18(4): 672-695.

[34] P. Papsiene, S. Vaitkevicius (2014); Human Resource Assessment Impact to Organization Climate: Case of Lithuanian Public Sector Organizations, Inzinerine Ekonomika - Engineering Economics, 25(2): 223-230.

[35] Y. Peng, G. Kou, G. Wang, Y. Shi (2011); FAMCDM: A Fusion Approach of MCDM Methods to Rank Multiclass Classification Algorithms. Omega, 39(6): 677-689.

[36] B. Rouyendegh, T. Erkan (2013); An Application of the fuzzy ELECTRE method for academic staff selection. Human Factors and Ergonomics in Manufacturing 83 Service Industries, 23(2): 107-115.

[37] B. Rouyendegh, T. Erkan (2012); Selection of academic staff using the fuzzy analytic hierarchy process (FAHP): a pilot study. Tehnicki vjesnik-Technical Gazette, 19(4): 923-929.

[38] A. Salehi, M. Izadikhah (2014); A novel method to extend SAW for decision-making problems with interval data, Decision Science Letters, 3(2): 225-236.

[39] G. Tzeng, J. Huang (2011); Multi Attribute Decision Making: Methods and Applications, CRCPress.

[40] D. Yu, W. Zhang, Y. Xu (2013); Group decision making under hesitant fuzzy environment with application to personnel evaluation, Knowledge-Based Systems 52: 1-10.

[41] E.K. Zavadskas, Z. Turskis, J. Tamošaitiene, V. Marina (2008); Multicriteria selection of project managers by applying grey criteria, Technological and Economic Development of Economy, 14(4): 462-477.

[42] E.K. Zavadskas, Z. Turskis (2011); Multiple criteria decision making (MCDM) methods in economics: an overview. Technological and economic development of economy, 17(2): 397427.

[43] E.K. Zavadskas, Z. Turskis, S. Kildienė (2014); State of art surveys of overviews on MCDM/MADM methods. Technological and economic development of economy, 20(1): 165-179.

[44] S. Zhang, S. Liu (2011); A GRA-based intuitionistic fuzzy multi-criteria group decision making method for personnel selection. Expert Systems with Applications, 38(9): 1140111405 . 